\title{
@creative
}

\section{Cube-contractions in 3-connected quadrangulations}

\author{
Yusuke Suzuki * \\ Department of Mathematics, Niigata University, \\ 8050 Ikarashi 2-no-cho, Nishi-ku, Niigata, 950-2181, Japan
}

Received 4 October 2013, accepted 10 January 2016, published online 30 January 2016

\begin{abstract}
A 3-connected quadrangulation of a closed surface is said to be $\mathcal{K}_{3}^{\prime}$-irreducible if no face- or cube-contraction preserves simplicity and 3-connectedness. In this paper, we prove that a $\mathcal{K}_{3}^{\prime}$-irreducible quadrangulation of a closed surface except the sphere and the projective plane is either (i) irreducible or (ii) obtained from an irreducible quadrangulation $H$ by applying 4-cycle additions to $F_{0} \subseteq F(H)$ where $F(H)$ stands for the set of faces of $H$. We also determine $\mathcal{K}_{3}^{\prime}$-irreducible quadrangulations of the sphere and the projective plane. These results imply new generating theorems of 3-connected quadrangulations of closed surfaces.
\end{abstract}

Keywords: Quadrangulation, closed surface, generating theorem.

Math. Subj. Class.: 05C10

\section{Introduction}

In this paper, we only consider simple graphs which have no loops and no multiple edges. We denote the vertex set and the edge set of a graph $G$ by $V(G)$ and $E(G)$, respectively. We say that $S \subset V(G)$ is a cut of $G$ if $G-S$ is disconnected. In particular, $S$ is called a $k$-cut if $S$ is a cut with $|S|=k$. A cycle $C$ of $G$ is said to be separating if $V(C)$ is a cut. Similarly, a simple closed curve $\gamma$ on a closed surface $F^{2}$ is said to be separating if $F^{2}-\gamma$ is disconnected.

A quadrangulation $G$ of a closed surface $F^{2}$ is a simple graph cellularily embedded on the surface so that each face is quadrilateral; thus, a 2-path on the sphere is not a quadrangulation. We denote the set of faces of $G$ by $F(G)$ throughout the paper. For quadrangulations we consider applying three reductions, called a face-contraction, a 4-cycle removal

* This work was supported by JSPS Grant-in-Aid for Young Scientists (B) No. 24740056.

E-mail address: y-suzuki@math.sc.niigata-u.ac.jp (Yusuke Suzuki) 


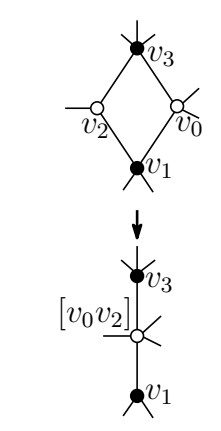

face-contraction

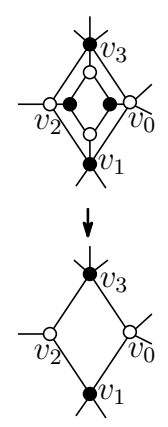

4-cycle removal

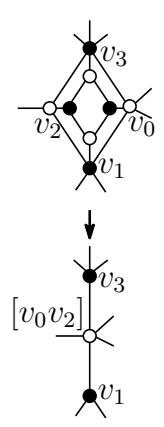

cube-contraction

Figure 1: Reductions for quadrangulations.

and a cube-contraction, as shown in Figure 1. (Precise definitions of these reductions will be given in the next section.) The corresponding inverse operations are called a vertexsplitting, a 4-cycle addition and a cube-splitting, respectively. In particular, the operations of a face-contraction and a 4-cycle removal were first introduced by Batagelj [1]

Irreducible quadrangulations, such that no face-contraction is applicable without making a loop or multiple edges, on a fixed closed surface with low genus were obtained in earlier papers. In [9], it was proven that a 4-cycle is the unique irreducible quadrangulation of the sphere, and that there exist precisely two irreducible quadrangulations of the projective plane shown in Figure 2, where $Q_{P}^{1}$ and $Q_{P}^{2}$ are the unique quadrangular embeddings of $K_{4}$ and $K_{3,4}$ on the projective plane, respectively. The irreducible quadrangulations of the torus and the Klein bottle have also been determined in [6, 5]. In [8], it was proven that for any closed surface $F^{2}$ there exist only finitely many irreducible quadrangulations of $F^{2}$, up to homeomorphism.

A 3-connected quadrangulation $G$ of a closed surface $F^{2}$ is said to be $\mathcal{K}_{3}$-irreducible if any of a face-contraction and a 4-cycle removal breaks simplicity or 3-connectedness of $G$. The following theorem is the starting point of the study of 3 -connected quadrangulations. (The definitions of a pseudo double wheel, a Möbius wheel and a double cube are given in the next section.)

Theorem 1.1 (Brinkmann et al.[2]). Any $\mathcal{K}_{3}$-irreducible quadrangulation of the sphere is isomorphic to a pseudo double wheel.

Observe that a 3-connected quadrangulation of the sphere corresponds to a 4-regular 3 -connected graph on the same surface by taking its dual. Broersma et al. [3] considered the same problem of the dual version with weaker conditions than those of Brinkmann. For the projective plane, Nakamoto proved the following.

Theorem 1.2 (Nakamoto[7]). Any $\mathcal{K}_{3}$-irreducible quadrangulation of the projective plane is isomorphic to either a Möbius wheel or $Q_{P}^{2}$.

Furthermore, the results in [4] imply the following. 
Theorem 1.3 (Nagashima et al.[4]). Let $G$ be a quadrangulation of a closed surface other than the sphere and the projective plane. Then $G$ is $\mathcal{K}_{3}$-irreducible if and only if $G$ is irreducible.
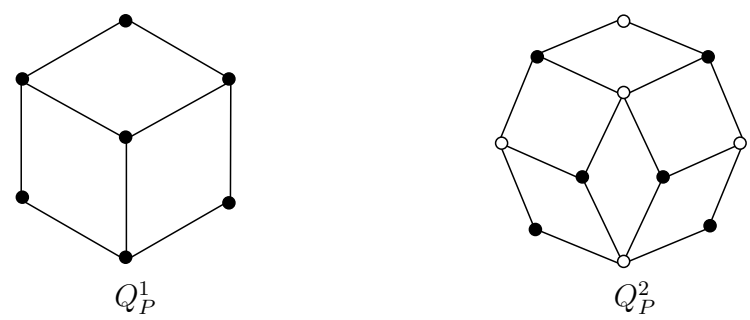

Figure 2: Irreducible quadrangulations on the projective plane.

In this paper, we determine other minimal subsets of 3-connected quadrangulations by replacing 4-cycle removals with cube-contractions. A 3-connected quadrangulation $G$ is said to be $\mathcal{K}_{3}^{\prime}$-irreducible if any of a face-contraction and a cube-contraction breaks the simplicity or the 3 -connectedness of $G$. The followings are our main results in the paper. In these statements, $F(H)$ stands for the set of faces of a quadrangulation $H$.

Theorem 1.4. Let $G$ be a $\mathcal{K}_{3}^{\prime}$-irreducible quadrangulation of a closed surface $F^{2}$ other than the sphere and the projective plane. Then, $G$ is either (i) irreducible or (ii) obtained from an irreducible quadrangulation $H$ by applying 4-cycle additions to $F_{0} \subseteq F(H)$.

Theorem 1.5. Let $G$ be a $\mathcal{K}_{3}^{\prime}$-irreducible quadrangulation of the sphere. Then, $G$ is either (i) a pseudo double wheel or (ii) a double cube.

Theorem 1.6. Let $G$ be a $\mathcal{K}_{3}^{\prime}$-irreducible quadrangulation of the projective plane. Then, $G$ is (i) a Möbius wheel, (ii) $Q_{P}^{2}$ or (iii) obtained from $Q_{P}^{1}$ (resp. $Q_{P}^{2}$ ) by applying 4-cycle additions to $F_{0} \subseteq F\left(Q_{P}^{1}\right)$ (resp. $F_{0} \subseteq F\left(Q_{P}^{2}\right)$ ).

Corollary 1.7. For any closed surface $F^{2}$, there exist only finitely many quadrangulations which are $\mathcal{K}_{3}^{\prime}$-irreducible but are not $\mathcal{K}_{3}$-irreducible, up to homeomorphism.

This paper is organized as follows. In the next section, we define the reductions used in this paper and introduce typical 3-connected quadrangulations on the sphere and the projective plane called a pseudo double wheel and a Möbius wheel, respectively. In Section 3, we develop some theoretical tools and prove Theorem 1.4. The last section is devoted to prove the planar case and the projective-planar case individually, using some figures.

\section{Reductions and typical quadrangulations}

Let $G$ be a quadrangulation of a closed surface $F^{2}$ and let $f$ be a face of $G$ bounded by a cycle $v_{0} v_{1} v_{2} v_{3}$. (We also use the notation like $f=v_{0} v_{1} v_{2} v_{3}$ in this paper.) The facecontraction of $f$ at $\left\{v_{0}, v_{2}\right\}$ in $G$ consists of identification of $v_{0}$ and $v_{2}$, and replacement of the resulting multiple edges $\left\{v_{0} v_{1}, v_{2} v_{1}\right\}$ and $\left\{v_{0} v_{3}, v_{2} v_{3}\right\}$ with two single edges, respectively. In the resulting graph, let $\left[v_{0} v_{2}\right]$ denote the vertex arisen by the identification of $v_{0}$ 
and $v_{2}$ (see the left-hand side of Figure 1). Similarly, we define the face-contraction of $f$ at $\left\{v_{1}, v_{3}\right\}$. The inverse operation of a face-contraction is called a vertex-splitting. We say that $f$ is contractible at $\left\{v_{0}, v_{2}\right\}$ in $G$, if the graph obtained from the face-contraction of $f$ at $\left\{v_{0}, v_{2}\right\}$ is simple. Assume in addition that $G$ is 3-connected. A face $f$ of $G$ is said to be 3 -contractible at $\left\{v_{0}, v_{2}\right\}$ if $f$ is contractible at $\left\{v_{0}, v_{2}\right\}$ and the graph obtained from the face-contraction is still 3 -connected.

Let $f=v_{0} v_{1} v_{2} v_{3}$ be a face of a quadrangulation $G$ of $F^{2}$. A 4-cycle addition to $f$ consists of inserting a 4-cycle $C=u_{0} u_{1} u_{2} u_{3}$ inside $f$ in $G$ and joining $v_{i}$ and $u_{i}$ for $i=0,1,2,3$. The inverse operation of a 4-cycle addition is called a 4 -cycle removal (of $C$ ), as shown in the center of Figure 1. We call the subgraph $Q$ isomorphic to a cube with eight vertices $u_{i}, v_{i}$ for $i=0,1,2,3$ an attached cube. For an attached cube $Q$, we call the above 4-cycle $C$ an inner 4-cycle of $Q$. In addition, we denote $\partial Q=v_{0} v_{1} v_{2} v_{3}$. Let $G$ be a 3 -connected quadrangulation of a closed surface having an attached cube $Q$. We say that an inner 4-cycle $C$ of $Q$ (or easily an attached cube $Q$ ) is removable if the graph obtained from $G$ by applying 4-cycle removal $C$ preserves the 3 -connectedness. (Observe that a 4-cycle removable never destroy simplicity of $G$.)

As mentioned in the introduction, there exist some results of 3-connected quadrangulations (or quadrangulations with minimum degree 3 ) on surfaces. In those results, the 4-cycle removal is necessary by the following reason: Let $\tilde{G}$ be the graph obtained from a 3-connected quadrangulation $G$ of a closed surface by applying 4-cycle additions to all faces of $G$. Clearly $\tilde{G}$ is 3 -connected, but we cannot apply any face-contraction to $\tilde{G}$ without creating a vertex of degree 2 .

Our third reduction of quadrangulations of closed surfaces is defined as a sequence of the above two reductions. Assume that a quadrangulation $G$ has an attached cube $Q$ with an inner 4-cycle $C$ and with $\partial Q=v_{0} v_{1} v_{2} v_{3}$. A cube-contraction of $Q$ at $\left\{v_{0}, v_{2}\right\}$ in $G$ consists of a 4-cycle removal of $C$ followed by a face-contraction at $\left\{v_{0}, v_{2}\right\}$ (see the right-hand side of Figure 1). The inverse operation of a cube-contraction is called a cubesplitting. We say that an attached cube $Q$ is contractible if the graph obtained from $G$ by applying a cube-contraction of $Q$ preserves the simplicity and the 3 -connectedness. One might suspect that if an attached cube $Q$ is contractible then $Q$ is removable (and the face that appeared by the removal is contractible). However, this is not true in general since a 4-cycle removal might break the 3 -connectedness of the graph.

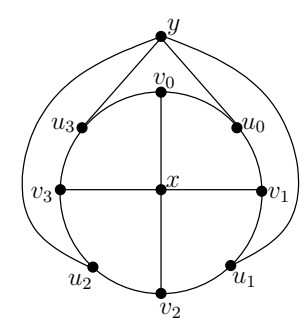

$W_{8}$

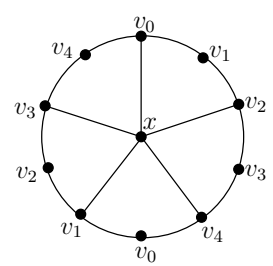

$\tilde{W}_{5}$

Figure 3: $W_{8}$ and $\tilde{W}_{5}$.

We need to describe two special types of embeddings. Firstly, embed a $2 k$-cycle $C=$ $v_{0} u_{0} v_{1} u_{1} \ldots v_{k-1} u_{k-1}(k \geq 3)$ into the sphere, put a vertex $x$ on one side and a vertex $y$ on 
the other side and add edges $x v_{i}$ and $y u_{i}$ for $i=0, \ldots, k-1$. The resulting quadrangulation of the sphere with $2 k+2$ vertices is said to be a pseudo double wheel and denoted by $W_{2 k}$ (see the left-hand side of Figure 3). The smallest pseudo double wheel is $W_{6}$, which is isomorphic to a cube, when the graphs are assumed to be 3-connected. The cycle $C$ of length $2 k$ is called the rim of $W_{2 k}$. We call a quadrangulation of the sphere obtained from $W_{6}$ by a single 4 -cycle addition a double cube, which is isomorphic to $C_{4} \times P_{2}$.

Secondly, embed a $(2 k-1)$-cycle $C=v_{0} v_{1} \ldots v_{2 k-2}(k \geq 2)$ into the projective plane so that the tubular neighborhood of $C$ forms a Möbius band. Next, put a vertex $x$ on the center of the unique face of the embedding and join $x$ to $v_{i}$ for all $i$ so that the resulting graph is a quadrangulation. The resulting quadrangulation of the projective plane with $2 k$ vertices is said to be a Möbius wheel and denoted by $\tilde{W}_{2 k-1}$ (see the right-hand side of Figure 3).

\section{Lemmas to prove Theorem 1.4}

The following lemma holds not only for quadrangulations but also for even embeddings of closed surfaces $F^{2}$, that is, for graphs embedded on $F^{2}$ with each face bounded by a cycle of even length. Taking a dual of an even embedding and using the odd point theorem, we can easily obtain this lemma.

Lemma 3.1. An even embedding of a closed surface has no separating closed walk of odd length.

Let $G$ be a quadrangulation of a closed surface $F^{2}$ and let $f=v_{0} v_{1} v_{2} v_{3}$ be a face of $G$. Then a pair $\left\{v_{i}, v_{i+2}\right\}$ is called a diagonal pair of $f$ in $G$, where the subscripts are taken modulo 4. A closed curve $\gamma$ on $F^{2}$ is said to be a diagonal $k$-curve for $G$ if $\gamma$ passes only through distinct $k$ faces $f_{0}, \ldots, f_{k-1}$ and distinct $k$ vertices $x_{0}, \ldots, x_{k-1}$ of $G$ such that for each $i, f_{i}$ and $f_{i+1}$ share $x_{i}$, and that for each $i,\left\{x_{i-1}, x_{i}\right\}$ forms a diagonal pair of $f_{i}$ of $G$, where the subscripts are taken modulo $k$.

Lemma 3.2. Let $G$ be a quadrangulation of a closed surface $F^{2}$ with a 2 -cut $\{x, y\}$. Then there exists a separating diagonal 2-curve for $G$ only through $x$ and $y$.

Proof. Observe that every quadrangulation of any closed surface $F^{2}$ is 2-connected and admits no closed curve on $F^{2}$ crossing $G$ at most once. Thus there exists a surface separating simple closed curve $\gamma$ on $F^{2}$ crossing only $x$ and $y$, since $\{x, y\}$ is a cut of $G$.

We shall show that $\gamma$ is a diagonal 2-curve. Suppose that $\gamma$ passes through two faces $f_{1}$ and $f_{2}$ meeting at two vertices $x$ and $y$. If $\gamma$ is not a diagonal 2-curve, then $x$ and $y$ are adjacent on $\partial f_{1}$ or $\partial f_{2}$. Since $G$ has no multiple edges between $x$ and $y$, and since $\{x, y\}$ is a 2 -cut of $G$, we may suppose that $x$ and $y$ are adjacent in $\partial f_{1}$, but not in $\partial f_{2}$. Here we can take a separating 3 -cycle of $G$ along $\gamma$. This contradicts Lemma 3.1.

Lemma 3.3. Let $G$ be a 3-connected quadrangulation of a closed surface $F^{2}$, and let $f=$ $v_{0} v_{1} v_{2} v_{3}$ be a face of $G$. If the face-contraction of $f$ at $\left\{v_{0}, v_{2}\right\}$ breaks 3 -connectedness of the graph but preserves simplicity, then $G$ has a separating diagonal 3-curve passing through $v_{0}, v_{2}$ and another vertex $x \in V(G)-\left\{v_{0}, v_{1}, v_{2}, v_{3}\right\}$.

Proof. Let $G^{\prime}$ be the quadrangulation of $F^{2}$ obtained from $G$ by the face-contraction of $f$ at $\left\{v_{0}, v_{2}\right\}$. Since $G^{\prime}$ has connectivity $2, G^{\prime}$ has a 2 -cut. By Lemma 3.2, $G^{\prime}$ has a separating diagonal 2-curve $\gamma^{\prime}$ passing through two vertices of the 2-cut. Clearly, one of the two 
vertices must be $\left[v_{0} v_{2}\right]$ of $G^{\prime}$, which is the image of $v_{0}$ and $v_{2}$ by the face-contraction of $f$. (Otherwise, $G$ would not be 3 -connected, a contradiction.) Let $x$ be a vertex of $G^{\prime}$ on $\gamma^{\prime}$ other than $\left[v_{0} v_{2}\right]$. Note that $x$ is not a neighbor of $\left[v_{0} v_{2}\right]$ in $G^{\prime}$. Now apply the vertexsplitting of $\left[v_{0} v_{2}\right]$ to $G^{\prime}$ to recover $G$. Then a diagonal 3-curve for $G$ passing through only $v_{0}, v_{2}$ and $x$ arises from $\gamma^{\prime}$ for $G^{\prime}$.

The next lemma plays an important role in a later argument.

Lemma 3.4. Let $G$ be a 3-connected quadrangulation on a closed surface $F^{2}$. If $G$ has a separating 4-cycle $C=x_{0} x_{1} x_{2} x_{3}$ and a face $f$ of $G$ such that

(i) one of the diagonal pairs of $f$ is $\left\{x_{i}, x_{i+2}\right\}$ for some $i$, and

(ii) $f$ has a separating diagonal 3-curve $\gamma$ intersecting $C$ only at $x_{i}$ and $x_{i+2}$ transversely,

then there exists a 3-contractible face in $G$.

Proof. Suppose that $G$ has a separating 4-cycle $C=x_{0} x_{1} x_{2} x_{3}$ and a face $f$ bounded by $a x_{1} c x_{3}$. Since $C$ is separating, $G$ has two subgraphs $G_{R}$ and $G_{L}$ such that $G_{R} \cup G_{L}=G$ and $G_{R} \cap G_{L}=C$. Suppose that $f$ is contained in $G_{R}$. Furthermore, we assume that $G_{R}$ contains as few vertices of $G$ as possible.

Since $C$ is separating, we have $\partial f \neq C$. By (ii), $f$ has a separating diagonal 3-curve $\gamma$ through $x_{1}, x_{3}$ and some vertex $x$. Note that $x \in V\left(G_{L}\right)-V(C)$ by the condition (ii) in the lemma. Now assume that $f$ is not 3-contractible at $\{a, c\}$. Observe that $\gamma$ (or the 3 -cut $\left\{x_{1}, x, x_{3}\right\}$ ) separates $a$ from $c$. Further, $G$ does not have both of edges $a x$ and $c x$ since $\partial f \neq C$. Therefore, there is no path of $G$ of length at most 2 joining $a$ and $c$ other than $a x_{1} c$ and $a x_{3} c$. Moreover, if $\{a, c\} \cap\left\{x_{0}, x_{2}\right\}=\emptyset$, then $f$ has no separating diagonal 3 -curve joining $a$ and $c$. This contradicts our assumption by Lemma 3.3 and so we may suppose that $a=x_{0}$ and $c \neq x_{2}$, and $f$ has a separating diagonal 3-curve, say $\gamma^{\prime}$, through $a\left(=x_{0}\right)$ and $c$.

Since $\gamma^{\prime}$ separates $x_{1}$ and $x_{3}$ and since $x_{2}$ is a common neighbors of $x_{1}$ and $x_{3}, \gamma^{\prime}$ must pass through $x_{2}$, and hence we can find a face $f^{\prime}$ of $G_{R}$ one of whose diagonal pair is $\left\{c, x_{2}\right\}$. Let $C^{\prime}$ be the 4-cycle $x_{1} x_{2} x_{3} c$ of $G$. Since $\operatorname{deg}(c) \geq 3$, we have $\partial f^{\prime} \neq C^{\prime}$, and hence $C^{\prime}$ is a separating 4-cycle in $G_{R}$ such that $C^{\prime} \neq C$. Moreover, $\gamma^{\prime}$ and $C^{\prime}$ cross transversely at $x_{2}$ and $c$. Therefore, $C^{\prime}$ and $f^{\prime}$ are a 4-cycle and a face which satisfy the assumption of the lemma, and moreover, $C^{\prime}$ can cut a strictly smaller graph than $G_{R}$ from $G$. Therefore, this contradicts the choice of $C$.

Lemma 3.5. Let $G$ be a 3-connected quadrangulation of a closed surface $F^{2}$. If $G$ is $\mathcal{K}_{3}$-irreducible then $G$ is $\mathcal{K}_{3}^{\prime}$-irreducible.

Proof. Let $G$ be a 3-connected quadrangulation of a closed surface. Assume that $G$ is not $\mathcal{K}_{3}^{\prime}$-irreducible. Then, $G$ has either a 3 -contractible face or a contractible cube. If $G$ has a 3 -contractible face, then $G$ is not $\mathcal{K}_{3}$-irreducible. Therefore, we suppose that $G$ has no 3 -contractible face but has a contractible cube $Q$ with an inner 4-cycle $C$ in the following argument.

Now, we apply a 4-cycle removal of $C$ to $G$ and let $G^{\prime}$ be the resulting quadrangulation. Let $f^{\prime}=\partial Q$ be the new face of $G^{\prime}$ into which $C$ was inserted. If $G^{\prime}$ is 3-connected, $G$ is not $\mathcal{K}_{3}$-irreducible by the definition, and we are done. Therefore, we assume that $G^{\prime}$ is not 3 -connected. By Lemma 3.2, there is a diagonal 2-curve $\gamma$ passing through $f^{\prime}$ and another 
face $f^{\prime \prime}$; otherwise, $G$ would have a 2 -cut, contrary to our assumption. Note that $f^{\prime \prime}$ is also a face in $G$. Now $\partial Q$ and $f^{\prime \prime}$ satisfy the conditions of Lemma 3.4, and hence there exists a 3 -contractible face in $G$. However, this contradicts the above assumption. Thus, the lemma follows.

In the following argument, we denote the set of $\mathcal{K}_{3}$-irreducible (resp. $\mathcal{K}_{3}^{\prime}$-irreducible) quadrangulations of a closed surface $F^{2}$ by $\mathcal{K}_{3} \mathcal{I}\left(F^{2}\right)\left(\right.$ resp. $\mathcal{K}_{3}^{\prime} \mathcal{I}\left(F^{2}\right)$ ).

Lemma 3.6. Let $G$ be a 3-connected quadrangulation of $F^{2}$. If $G \in \mathcal{K}_{3}^{\prime} \mathcal{I}\left(F^{2}\right) \backslash \mathcal{K}_{3} \mathcal{I}\left(F^{2}\right)$, then $G$ has an attached cube $Q$ such that the graph obtained from $G$ by applying a 4 -cycle removal of $Q$ is in $\mathcal{K}_{3}^{\prime} \mathcal{I}\left(F^{2}\right)$.

Proof. Let $G$ be in $\mathcal{K}_{3}^{\prime} \mathcal{I}\left(F^{2}\right) \backslash \mathcal{K}_{3} \mathcal{I}\left(F^{2}\right)$. By the definition, $G$ has an attached cube $Q$ with an inner 4-cycle $C$ which is removable, but is not contractible. We apply a 4-cycle removal of $C$ and let $G^{-}$be the resulting quadrangulation. We denote the new face of $G^{-}$by $f^{-}$, where $f^{-}=\partial Q$.

First, we confirm that $G^{-}$is 3 -connected. Otherwise, $G^{-}$has a 2 -cut and has a separating diagonal 2-curve $\gamma$ on $F^{2}$ by Lemma 3.2. If $\gamma$ does not pass through $f^{-}$then $\gamma$ would also be a diagonal 2-curve in $G$, a contradiction. Let $f_{0}$ be the other face passed by $\gamma$. Here, $f_{0}$ and $\partial Q$ in $G$ satisfy the conditions in Lemma 3.4 and there exists a 3-contractible face, contrary to $G$ being $\mathcal{K}_{3}^{\prime}$-irreducible.

By way of contradiction, assume that $G^{-}$is not in $\mathcal{K}_{3}^{\prime} \mathcal{I}\left(F^{2}\right)$. That is, $G^{-}$has either (a) a 3-contractible face or (b) a contractible cube. First, we assume (a) and let $f$ be a 3 -contractible face in $G^{-}$. If $f^{-}=f$, the attached cube $Q$ in $G$ would be contractible, contrary to $G$ being $\mathcal{K}_{3}^{\prime}$-irreducible. Thus, suppose $f^{-} \neq f$. In this case, let $G^{\prime}$ be the resulting 3-connected quadrangulation after applying a face-contraction of $f$ in $G^{-}$. Since any 4-cycle addition doesn't break the 3-connectedness of a quadrangulation, the graph obtained from $G^{\prime}$ by a 4 -cycle addition to $f^{-}$is clearly 3 -connected. This means that $f$ is also 3-contractible in $G$, a contradiction.

Next, suppose (b) and let $Q^{\prime}$ be such a contractible cube with $\partial Q^{\prime}=v_{0} v_{1} v_{2} v_{3}$. If $Q^{\prime}$ does not contain $f^{-}$as one of its five faces, $Q^{\prime}$ is also contractible in $G$ and $G$ would not be $\mathcal{K}_{3}^{\prime}$-irreducible by the similar argument as above. Thus, we assume that $Q^{\prime}$ contains $f^{-}$. Let $C=u_{0} u_{1} u_{2} u_{3}$ denotes the inner 4-cycle of $Q^{\prime}$ where $u_{i} v_{i} \in E\left(Q^{\prime}\right)$ for $i=$ $0,1,2,3$. We consider the following two cases up to symmetry; (b-1) $f^{-}=C$ and (b2) $f^{-}=v_{0} u_{0} u_{1} v_{1}$. At first, suppose (b-1). Here, we apply a face-contraction of $f_{1}=$ $v_{0} u_{0} u_{1} v_{1}$ at $\left\{u_{0}, v_{1}\right\}$ to $G$. If the above face-contraction breaks the 3 -connectedness of $G$, there exists a face $f_{2}=v_{1} x v_{3} y$ in the outside of $Q^{\prime}$ by Lemma 3.3; note that it clearly preserves the simplicity of the graph since $v_{1} \neq v_{3}$. Now, a separating diagonal 3 -curve passing through $\left\{v_{1}, u_{0}, v_{3}\right\}$ satisfies the conditions of Lemma 3.4 and hence $G$ is not $\mathcal{K}_{3}^{\prime}$ irreducible, contrary to our assumption. In fact, an analogous proof is valid for (b-2) if we try to apply a face contraction at $\left\{v_{1}, u_{2}\right\}$ to $G$. Therefore the lemma follows.

Lemma 3.7. Let $G$ be a 3-connected quadrangulation of a closed surface $F^{2}$. If $G \in$ $\mathcal{K}_{3}^{\prime} \mathcal{I}\left(F^{2}\right) \backslash \mathcal{K}_{3} \mathcal{I}\left(F^{2}\right)$, then $G$ can be obtained from $H \in \mathcal{K}_{3} \mathcal{I}\left(F^{2}\right)$ by applying 4-cycle additions to $F_{0} \subseteq F(H)$.

Proof. Assume that $G \in \mathcal{K}_{3}^{\prime} \mathcal{I}\left(F^{2}\right) \backslash \mathcal{K}_{3} \mathcal{I}\left(F^{2}\right)$. By the previous lemma, there exists a sequence of $\mathcal{K}_{3}^{\prime}$-irreducible quadrangulations $G=G_{0}, G_{1}, \ldots, G_{k}$ such that $G_{i+1}$ is obtained from $G_{i}$ by a single 4 -cycle removal of $C_{i}$, where $G_{k} \in \mathcal{K}_{3} \mathcal{I}\left(F^{2}\right)$. (Since the 
number of vertices of $G$ is finite, $G_{k} \in \mathcal{K}_{3} \mathcal{I}\left(F^{2}\right)$.) Let $Q_{i}$ denote an attached cube in $G_{i}$ with an inner 4-cycle $C_{i}$.

For a contradiction, we assume that there exists $l \in\{0, \ldots, k-2\}$ such that $G_{l}$ is obtained from $G_{l+1}$ by a 4-cycle addition which is put on a face not of $F\left(G_{k}\right)$; this $l$ should be maximal. This implies that $C_{l}$ is put on a face of $Q_{l+1}$ as one of its five faces. Then the same argument as the proof of Lemma 3.6 holds and hence $G_{l}$ would not be $\mathcal{K}_{3}^{\prime}$ irreducible, contrary to our assumption. Thus for each $i \in\{0, \ldots, k-1\}, G_{i}$ is obtained from $G_{i+1}$ by a 4 -cycle addition which is put on a face of $F\left(G_{k}\right)$.

Proof of Theorem 1.4. By Lemma 3.5, we have $\mathcal{K}_{3} \mathcal{I}\left(F^{2}\right) \subseteq \mathcal{K}_{3}^{\prime} \mathcal{I}\left(F^{2}\right)$. Furthermore, by Theorem 1.3 and Lemma 3.7, we obtain (i) and (ii) in the statement. Thus, we have got a conclusion.

\section{Spherical and projective-planar cases}

In this section, we discuss the spherical case and the projective-planar case.

Proof of Theorem 1.5. Let $G$ be a $\mathcal{K}_{3}^{\prime}$-irreducible quadrangulation of the sphere. We have $\mathcal{K}_{3} \mathcal{I}\left(S^{2}\right) \subseteq \mathcal{K}_{3}^{\prime} \mathcal{I}\left(S^{2}\right)$ by Lemma 3.5 , where $S^{2}$ stands for the sphere.

If $G$ is $\mathcal{K}_{3}$-irreducible, then $G$ is isomorphic to a pseudo double wheel by Theorem 1.1. If $G$ is in $\mathcal{K}_{3}^{\prime} \mathcal{I}\left(S^{2}\right) \backslash \mathcal{K}_{3} \mathcal{I}\left(S^{2}\right), G$ can be obtained from a pseudo double wheel $W_{2 k}$ $(k \geq 3)$ by some 4 -cycle additions to faces of $W_{2 k}$ by Lemma 3.7. However if $k \geq 4$, $G$ has a 3-contractible face (or a contractible cube), as shown in the first operation in Figure 4. (For example, the entire Figure 4 presents a sequence of a face-contraction and a cube-contraction which deforms $W_{8}$ with an attached cube $Q$ into $W_{6}$, preserving the 3-connectedness.)

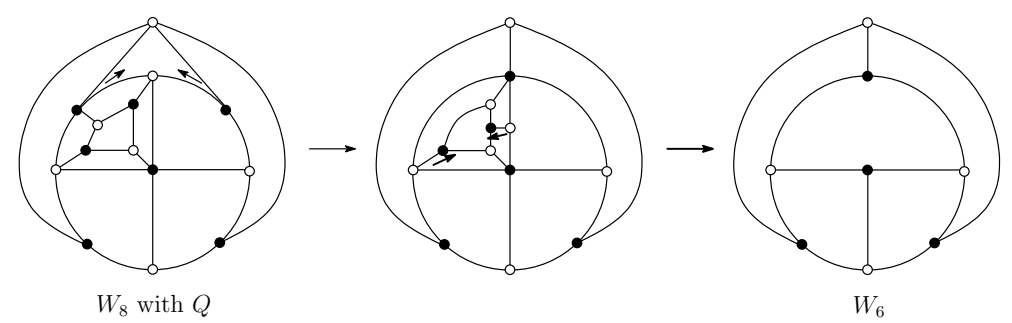

Figure 4: $W_{8}$ with an attached cube $Q$ deformed into $W_{6}$.

Therefore, we only consider the case of $k=3$ in the following argument. Assume that $G$ is obtained from $W_{6}$ by at least two 4-cycle additions to faces of $W_{6}$. Similarly to the above argument, $G$ would have a 3-contractible face (or a contractible cube), as in Figure 5, contrary to $G$ being $\mathcal{K}_{3}^{\prime}$-irreducible; note that it suffices to discuss these two cases, up to symmetry. Therefore, we conclude that $G$ is obtained from $W_{6}$ by exactly one 4-cycle addition. This is nothing but a double cube; observe that a double cube has no 3-contractible face and no contractible cube.

To conclude with, we prove the projective-planar case. 

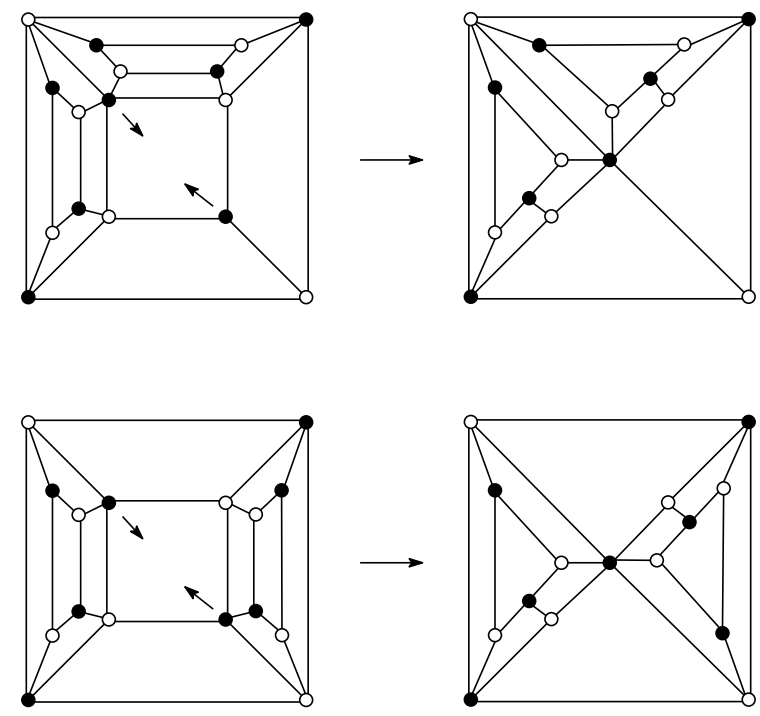

Figure 5: $W_{6}$ with two attached cubes can be reduced.

Proof of Theorem 1.6. In this case, we use Möbius wheels $\tilde{W}_{k}(k \geq 3)$ and $Q_{P}^{2}$ as base graphs by Theorem 1.2.

First we consider the former case. Similarly to the previous proof (and see Figure 6), we consider only a Möbius wheel $\tilde{W}_{3}$ as a base to which we apply some 4-cycle additions. However, $\tilde{W}_{3}\left(=Q_{P}^{1}\right)$ is isomorphic to the complete graph with four vertices, and hence it is irreducible. This fact implies that every $G$ obtained from $\tilde{W}_{3}$ by applying at most three 4-cycle additions is $\mathcal{K}_{3}^{\prime}$-irreducible since any face-contraction and any cube-contraction to $G$ destroys the simplicity of the graph, or results in a vertex of degree 2. From this case, we obtain exactly three quadrangulations in $\mathcal{K}_{3}^{\prime} \mathcal{I}\left(P^{2}\right) \backslash \mathcal{K}_{3} \mathcal{I}\left(P^{2}\right)$, up to homeomorphism, where $P^{2}$ stands for the projective plane.

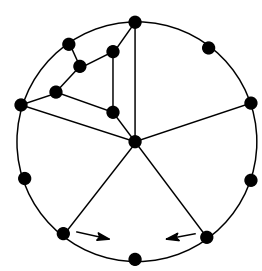

$\tilde{W}_{5}$ with $Q$
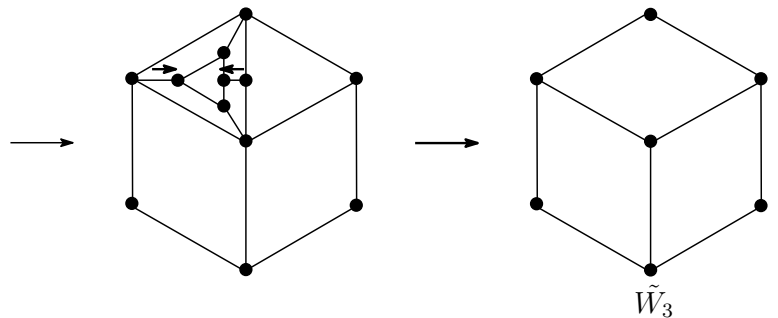

Figure 6: $\tilde{W}_{5}$ with an attached cube $Q$ deformed into $\tilde{W}_{3}$.

Similarly, as the latter case, we obtain the other ten quadrangulations in $\mathcal{K}_{3}^{\prime} \mathcal{I}\left(P^{2}\right) \backslash$ $\mathcal{K}_{3} \mathcal{I}\left(P^{2}\right)$ from $Q_{P}^{2}$; consider all the way to put attached cubes into faces of $Q_{P}^{2}$, up to symmetry. As a result, we have $\left|\mathcal{K}_{3}^{\prime} \mathcal{I}\left(P^{2}\right) \backslash \mathcal{K}_{3} \mathcal{I}\left(P^{2}\right)\right|=13$ in total. 


\section{References}

[1] V. Batagelj, An inductive definition of the class of 3-connected quadrangulations of the plane, Discrete Math. 78 (1989), 45-53, doi:10.1016/0012-365X(89)90159-3.

[2] G. Brinkmann, S. Greenberg, C. Greenhill, B. D. McKay, R. Thomas and P. Wollan, Generation of simple quadrangulations of the sphere, Discrete Math. 305 (2005), 33-54, doi:10.1016/j.disc. 2005.10.005.

[3] H. J. Broersma, A. J. W. Duijvestijn and F. Göbel, Generating all 3-connected 4-regular planar graphs from the octahedron graph, J. Graph Theory 17 (1993), 613-620, doi:10.1002/jgt. 3190170508.

[4] M. Nagashima, A. Nakamoto, S. Negami and Y. Suzuki, Generating 3-connected quadrangulations on surfaces, Ars Combin. 116 (2014), 371-384.

[5] A. Nakamoto, Irreducible quadrangulations of the Klein bottle, Yokohama Math. J. 43 (1995), 125-139.

[6] A. Nakamoto, Irreducible quadrangulations of the torus, J. Combin. Theory Ser. B 67 (1996), 183-201, doi:10.1006/jctb.1996.0040.

[7] A. Nakamoto, Generating quadrangulations of surfaces with minimum degree at least 3, J. Graph Theory 30 (1999), 223-234, doi:10.1002/(SICI)1097-0118(199903)30:3〈223::AID-JGT7 $\rangle .3$. CO;2-D.

[8] A. Nakamoto and K. Ota, Note on irreducible triangulations of surfaces, J. Graph Theory 20 (1995), 227-233, doi:10.1002/jgt.3190200211.

[9] S. Negami and A. Nakamoto, Diagonal transformations of graphs on closed surfaces, Sci. Rep. Yokohama Nat. Univ. Sect. I Math. Phys. Chem. (1993), 71-97. 